\title{
Dinâmica de implantação do Sales and Operations Planning: principais desafios
}

\section{Dynamic implementation of Sales and Operations Planning: main challenges}

\author{
Carolina Belotti Pedroso \\ Andrea Lago da Silva ${ }^{1}$
}

\begin{abstract}
Resumo: Existem vários desafios que as empresas enfrentam nos dias atuais, dentre eles a necessidade de construir maior sincronia entre demanda e suprimentos. O objetivo deste artigo é caracterizar a dinâmica do processo de S\&OP, identificando a motivação, os objetivos, os desafios e os principais resultados obtidos por empresas que já o adotaram no Brasil. Inicialmente realizou-se revisão de literatura e, a seguir, um estudo multicaso em três empresas brasileiras. Dentre as principais motivações, observou-se o alinhamento de informação (previsão de vendas e capacidade e maior conhecimento do mercado) entre as áreas. Dentre os objetivos estavam: melhorar o planejamento, em especial de capacidade, incluindo Vendas no processo. Os principais desafios foram: melhorar a comunicação fragmentada e, construir visibilidade da gestão de capacidade. Como resultado, observou-se melhoria na comunicação, otimização do planejamento de capacidades, maior assertividade na previsão de vendas. A principal contribuição foi identificar que a implementação do $\mathrm{S} \& O P$ exigirá que desafios diversos sejam enfrentados a fim de que o processo possa oferecer os resultados esperados para a gestão de operações.
\end{abstract}

Palavras-chave: Sales and Operations Planning; Gestão de operações; Gestão da demanda e gestão da cadeia de suprimentos.

\begin{abstract}
The need to build greater synchronization between the demand side and the supply side of the business is among the many challenges faced by a company. The objective of this paper is to characterize the dynamics of the $S \& O P$ process by identifying the motivation, goals, challenges and results achieved by organizations that have adopted these processes in Brazil to promote this synchronization. To this end, a systematic literature review followed by a multi-case study on three Brazilian organizations were carried out. The initial objective was to improve the planning, in particular the capacity, while including Sales in the process. The main challenges were to improve the fragmented communication process and build visibility regarding capacity management across the organization. Improvement in communication, optimization of capacity planning and greater assertiveness in sales forecasting were some of the results of the $S \& O P$ process implementation. The main contribution of this research was the identification of the idea that $S \& O P$ implementation requires the facing of several challenges so that the process can deliver the expected results for operations management.
\end{abstract}

Keywords: Sales and Operations Planning; Operations management; Demand management and supply chain managements.

\section{Introdução}

O processo de S\&OP teve sua origem na década de 1950, a partir do planejamento agregado de produção, evoluindo para um processo de negócio mais amplo (Singhal \& Singhal, 2007), conhecido como Manufacturing Resource Planning (MRP II), popularizado na década de 1980 (Wallace \& Stahl, 2003; Dougherty \& Gray, 2006; Thomé et al., 2012b). As aplicações iniciais ocorreram, na sua maioria, em indústrias (Grimson \& Pyke, 2007), sendo que atualmente esta tendência continua presente, havendo publicações sobre o assunto predominantemente na área de manufatura (Gianesi, 1998; Ivert \& Jonsson, 2010).

Sales and Operations Planning (S\&OP) pode ser conceituado como um processo liderado pela alta gerência, que possui a responsabilidade de avaliar e revisar projeções, considerando o tempo para demanda, suprimentos, produtos, projetos estratégicos e planos financeiros, tendo como finalidade alinhar demanda e suprimentos. Trata-se de um processo de planejamento

\footnotetext{
${ }^{1}$ Departamento de Engenharia de Produção, Universidade Federal de São Carlos - UFSCar, Rod. Washington Luís, Km 235 , CEP 13565-905, São Carlos, SP, Brasil, e-mail: carolina.belotti@usp.br; deialago@ufscar.br
}

Recebido em Out. 9, 2014 - Aceito em Maio 5, 2015

Suporte financeiro: CAPES e Fundação de Amparo à Pesquisa do Estado de São Paulo - FAPESP (Processo n²011/06008-8). 
conjunto com tomada de decisão que realinha os planos táticos das áreas funcionais da empresa a fim de auxiliar a organização no alcance de metas e objetivos do negócio (Palmatier \& Crum, 2010; Lapide, 2011). O processo de S\&OP, geralmente, acontece no decorrer de um mês (mensal, com horizonte de planejamento de 24 meses), sendo fundamentado em processos de planejamento tático, com a finalidade de balancear demanda e suprimentos, assegurando que os planos de todas as funções organizacionais estejam alinhados, de acordo com a estratégia do negócio (Ivert \& Jonsson, 2010). Uma das principais metas do S\&OP é facilitar o planejamento mestre da produção, planejamento da demanda e o fluxo de informações entre eles (Oliva \& Watson, 2011).

\subsection{Objetivo e justificativa}

Neste artigo, busca-se explorar uma visão mais aprofundada do processo de S\&OP, já que pesquisas sobre o tema são escassas no Brasil (Santos, 2006; Bremer et al., 2008a,b; Pandim et al., 2012; Thomé et al., 2012a,b), principalmente estudos que contenham dados empíricos que os sustentem (Ivert et al., 2015). O objetivo deste artigo é caracterizar a dinâmica do processo de S\&OP, identificando o contexto, a motivação/objetivos, os desafios e os principais resultados obtidos por empresas que possuem como estratégia de resposta a demanda Make to Stock (MTS) e estão estabelecidas no Brasil.

Apesar do crescente número de empresas que vêm adotando o processo de $\mathrm{S} \& \mathrm{OP}$, algumas empresas não estão obtendo os benefícios esperados. De acordo com uma pesquisa, conduzida por interesse das empresas IBM e Oracle, por volta de um terço dos programas de S\&OP em empresas americanas falha ou traz resultados fracos (pesquisa realizada pela "Supply Chain Management Review" citada por Singh, 2010). Estes resultados são atribuídos à inexistência de fatores de sucesso que garantam a implementação bem-sucedida do S\&OP, como apoio da alta gerência, integração interfuncional, treinamentos e, entendimento do processo, dentre outros. Observa-se que a implementação do processo de S\&OP pode ser caracterizada como complexa (Grimson \& Pyke, 2007; Viswanathan, 2011), o que torna relevante o entendimento da dinâmica do processo.

\subsection{Estrutura do artigo}

Este artigo se estrutura em 5 seções. Na seção 2, é feita uma revisão de literatura e é mostrada uma visão geral do processo de S\&OP, a definição das principais motivações para sua implantação, principais objetivos e principais resultados alcançados. Na seção 3 , são apresentados os principais procedimentos metodológicos. Na seção 4, são apresentados e discutidos os resultados dos estudos de caso, seguidos da análise intercasos, contendo principais resultados obtidos e desafios enfrentados. Na seção 5 , são apresentadas as principais conclusões. Por último, têm-se as referências e Apêndices 1 e 2 com protocolo e questionário utilizados

\section{Revisão de literatura}

\subsection{Visão geral do processo de S\&OP}

O processo de S\&OP reconcilia, ajusta e comunica o plano da companhia, ligando planos operacionais e financeiros e transformando-os em um conjunto de números únicos. Tal feito é possível por meio da realização de reuniões contínuas e regulares, que buscam envolver todos os participantes no processo que estão alocados nas variadas áreas funcionais da organização (APICS, 2009; Wallace, 2001). A ideia é conciliar os planos de suprimento, de demanda e de novos produtos, tanto no nível detalhado quanto no nível agregado, e verificar sua aderência ao plano de negócio (APICS, 2009). O processo interliga os planos de Vendas, Marketing, Desenvolvimento, Manufatura, Compras e Finanças em um conjunto integrado de planos (Cox \& Blackstone, 2002) e facilita a comunicação, compartilhamento de informações e o planejamento entre áreas voltadas ao mercado como Vendas e Marketing e áreas como Manufatura e a Cadeia de Suprimentos (Esper et al., 2010).

O processo de S\&OP possui algumas características principais, podendo-se citar: processo de planejamento de caráter tático, integrado e interfuncional na empresa; possui a capacidade de integrar os planos da organização em um único plano; seu horizonte de planejamento gira em torno do médio prazo, conectando o nível estratégico ao nível operacional; e promove a criação de valor que está associada ao desempenho da empresa (Thomé et al., 2012b). O processo integra a empresa tanto no nível vertical quanto no nível horizontal. A integração, que ocorre em âmbito vertical, ocorre entre os variados níveis de decisão, objetivando assegurar que as decisões tomadas em nível estratégico (perspectiva de longo prazo) sejam implementadas em nível operacional. Desta forma, o processo atua como elo entre as reuniões de planejamento estratégico da alta gerência e as decisões gerenciais tomadas no dia a dia da Produção e Operações. A integração horizontal ocorre entre decisões tomadas em um mesmo nível, envolvendo diferentes departamentos da empresa, tais como Marketing, manufatura e finanças, por exemplo. O processo liga funções distintas, assegurando que haja uma única direção dos objetivos da empresa (Côrrea et al., 2007).

Embora o interesse no assunto tenha ganhado força nas décadas passadas, a literatura sobre o assunto ainda é dispersa (Thomé et al., 2014). Além disso, 
apesar de o S\&OP parecer de fácil entendimento, as organizações têm dificuldade de implementá-lo e de alcançar os benefícios esperados. Observa-se, deste modo, falta de orientações sobre a implementação do processo (Tuomikangas \& Kaipia, 2014). São variados os estudos sobre S\&OP na literatura, porém ainda são limitadas as abordagens sobre a caracterização do processo como um todo. No geral, os autores exploram diferentes vertentes como: temas associados ao S\&OP e suas interações (Olhager et al., 2001; Feng et al., 2008; Wang \& Hsu, 2010); módulos de expansão que apoiam o S\&OP (Burrows, 2007; Ivert \& Jonsson, 2010, 2014; Baumann, 2010; VICS, 2010); exploram de forma teórica o processo evolutivo da aplicação do processo de S\&OP (Palmatier \& Crum, 2010; Sheldon, 2006; Cecere et al., 2009; Ling \& Coldrick, 2009; VICS, 2010); estudam o cenário das empresas no que diz respeito ao estágio evolutivo do S\&OP (Wing \& Perry, 2001; Lapide, 2005; Grimson \& Pyke, 2007; Feng et al., 2008; Viswanathan, 2011; Cecere et al., 2009); estudam alguns dos fatores estratégicos de sucessos para implantação do S\&OP (Muzumdar \& Fontanella, 2006; Wallace, 2001, 2004; Wing \& Perry, 2001; Lapide, 2004; Lapide, 2005; Dougherty \& Gray, 2006; Elbaum, 2005; Sharp, 2006; Sheldon, 2006; Corrêa et al., 2007; Grimson \& Pyke, 2007; Tearnan, 2008; Boyer, 2009; Cecere et al., 2009; VICS, 2010); ou ainda, estudam algumas barreiras que dificultam a implantação do processo de S\&OP (Gilmore, 2005; Bower, 2005; Corrêa et al., 2007; Grimson \& Pyke, 2007; Moon, 2006; Cecere et al., 2009).

Verifica-se que este se trata de um campo de estudo ainda pouco explorado no Brasil, sendo estudado principalmente nos Estados Unidos, seu local de origem e na Europa, de modo geral. Destaca-se também que o processo passou por modificações ao longo de sua existência, uma vez que as necessidades das empresas mudaram ao longo do tempo, alterando seus objetivos. De modo inicial, o processo era "reativo", sendo que seus objetivos tinham como foco o desenvolvimento de planos operacionais. Mais recentemente, o processo de S\&OP passou a ser "antecipativo" e as metas do processo passaram a cuidar do equilíbrio entre demanda e suprimentos. Uma nova modificação do processo surgiu, trazendo um processo "colaborativo" no qual seus objetivos eram a lucratividade. Atualmente o processo se caracteriza como "orquestrativo" e tem como foco a demanda, analisando trade offs a fim de gerenciar a demanda e obter respostas de forma otimizada (Cecere et al., 2009).

\subsection{Principais motivações}

O Sales and Operations Planning (S\&OP) se configura como um processo eficaz para as organizações, oferecendo meios para reagir rapidamente às mudanças. $\mathrm{O}$ processo de $\mathrm{S} \& \mathrm{OP}$ surge no intuito de auxiliar as empresas a gerenciar e diminuir as mudanças vivenciadas no ambiente de negócios, levando a uma cadeia de suprimentos mais alinhada (Sheldon, 2006; Baumann, 2010). O objetivo nos dias atuais é desenvolver sincronia entre demanda e suprimento, buscando equilíbrio entre volume e mix. Para tal, é necessário que haja um processo capaz de gerenciar estes fluxos de maneira eficaz, garantindo a entrega dos produtos aos clientes no prazo e nas condições acordadas (Wallace, 2001). Uma das principais metas do $\mathrm{S} \& \mathrm{OP}$ é facilitar o planejamento mestre da produção, planejamento da demanda e o fluxo de informações entre eles (Oliva \& Watson, 2011). O processo de S\&OP visa elaborar planos de operações e financeiros que estejam em um consenso único. Isto é possível através de reuniões realizadas de modo coordenado, conduzidas pela alta gerência a fim de integrar planos estratégicos, operacionais e financeiros em um horizonte de tempo de longo prazo (VICS, 2010). Ademais, o processo é capaz de unir diferentes metas da organização em um plano único (Tudorie \& Borangiu, 2011).

Processos de S\&OP devidamente implementados, e que contenham fatores estratégicos para seu sucesso, podem levar a cadeia de suprimentos a um desempenho operacional responsivo (Lapide, 2004). O S\&OP trabalha como um processo de planejamento colaborativo, integrando áreas funcionais da organização e apresenta, como uma de suas prioridades, facilitar o planejamento de produção, o planejamento de demanda e a transmissão do fluxo de informações presente entre ambos. O S\&OP torna a transferência dessas informações mais fácil, promovendo não somente uma sincronização dos planejamentos de demanda e de produção, mas também oferece planejamentos mais sofisticados (Oliva \& Watson, 2009). Desta forma, a equipe de S\&OP deve ser interfuncional, devido a conflitos funcionais entre as áreas de Vendas, Marketing, Logística, Finanças e Operações. Tais conflitos ocorrem devido a problemas estruturais da organização, tais como sistemas de recompensa e de avaliação contraditórios, complexidades relativas à produção, a produtos e à orientação de mercado (Thomé et al., 2012b). O processo deve incluir representantes das seguintes áreas: Vendas e Marketing, executando o gerenciamento e a previsão de demanda; Operações, responsável pela aquisição de suprimentos, gestão de estoques, operações da cadeia de suprimentos, e plano mestre de produção; e Finanças, contribuindo com atividades de caráter financeiro (Grimson \& Pyke, 2007).

\subsection{Principais objetivos}

O processo de S\&OP tem como objetivo balancear demanda e suprimentos em nível de volume e mix. $\mathrm{O}$ volume se refere aos índices globais de vendas, famílias de produtos de modo geral, taxas de produção, inventários agregados e pedidos que se encontram 
pendentes ou em situação de atraso. Por sua vez, o mix diz respeito a produtos específicos, sequência de produção e pedidos de clientes. Caso o volume seja controlado de forma efetiva haverá problemas de menor escala com respeito ao mix, porém, se o volume carecer de um planejamento bem elaborado, os problemas com mix crescerão (Wallace, 2001). Os principais objetivos do S\&OP foram sintetizados no Quadro 1.

\subsection{Principais resultados obtidos com S\&OP}

Os benefícios da adoção do processo de S\&OP são amplos em muitas organizações. Em primeiro lugar, pode-se melhorar o desempenho operacional, incluindo entregas realizadas a tempo (OTIF - On Time in Full), redução de inventário, melhorias na qualidade e maximização de lucros. Na medida em que ganham mais experiência com a realização dos ciclos de S\&OP, as organizações começam a melhorar seu desempenho, fato que leva à melhoria do desempenho do negócio como um todo, contribuindo para os resultados oferecidos aos grupos de interesse da organização. Observa-se também que com o S\&OP a equipe de gestão se torna mais eficaz no gerenciamento do negócio (Palmatier \& Crum, 2010).

Dentre os principais resultados derivados do S\&OP, encontra-se a capacidade de obtenção e retenção do equilíbrio entre demanda e suprimento, sendo capaz, inclusive, de envolver toda a cadeia produtiva (Wallace, 2004). No mesmo sentido, a integração da cadeia de suprimentos com clientes e fornecedores é considerada um aspecto-chave do processo de S\&OP (Thomé et al., 2012b, 2014). O processo de S\&OP objetiva, ainda, atar visões do futuro à situação vivenciada no presente, ocasionando planejamentos contínuos, a fim de sanar quaisquer fontes de desequilíbrio, como variações de demanda e disponibilidade de recursos (Côrrea et al., 2007; Mellen et al., 2010).

O resultado esperado do processo é a sincronia entre demanda, suprimentos e o plano financeiro em um horizonte de tempo de 18 a 24 meses. Busca-se identificar riscos e oportunidades, além de desenvolver planos de ação para cobrir quaisquer lacunas no plano anual da empresa, assim como no plano estratégico de longo prazo (VICS, 2010). Caso o processo de S\&OP seja devidamente conduzido, a empresa será capaz de ligar o plano estratégico da companhia com sua execução, revisar as métricas de desempenho a fim de obter melhoria contínua (Cox \& Blackstone, 2002) e oferecer um processo de planejamento integrado do negócio com potencial de alinhar planos operacionais e estratégicos, de acordo com os objetivos financeiros da empresa (Baumann, 2010). Decisões realizadas em tempo correto, objetivando a melhor combinação de

Quadro 1. Principais objetivos do S\&OP.

\begin{tabular}{|c|c|}
\hline Principais objetivos do S\&OP & Autores \\
\hline $\begin{array}{l}\text { Elaborar e revisar planos operacionais e o desempenho da empresa para um horizonte } \\
\text { de tempo de, em média, dois (2) anos. }\end{array}$ & Basu (2001) \\
\hline $\begin{array}{l}\text { Equilibrar demanda e suprimentos em nível de volume. } \\
\text { Avaliar o desempenho da empresa de forma contínua; alinhar as metas da empresa } \\
\text { ao nível operacional; desenvolver planos confiáveis e realísticos de demanda e de } \\
\text { suprimentos a fim de alinhar demanda e suprimentos e promover melhorias na cadeia, } \\
\text { reduzindo os custos da empresa. } \\
\text { Apoiar o planejamento estratégico da organização, ligando o plano estratégico da } \\
\text { empresa ao nível operacional; garantir que os planos elaborados sejam condizentes } \\
\text { com a realidade; assegurar que as mudanças sejam realizadas de modo satisfatório; } \\
\text { oferecer um bom nível de serviço ao cliente; colaborar com a avaliação de desempenho; } \\
\text { promover espírito de grupo, por meio de trabalho conjunto. }\end{array}$ & $\begin{array}{c}\text { Wallace (2001) } \\
\text { Bower (2005) } \\
\text { Corrêa et al. (2007) }\end{array}$ \\
\hline $\begin{array}{l}\text { Desenvolver e delimitar metas de Produção e de Vendas, por meio de reuniões de rotina. } \\
\text { Disponibilizar o produto final ao consumidor com o menor custo possível, bem como } \\
\text { demais recursos empregados. } \\
\text { Promover consenso entre diferentes funções da empresa, criando um único conjunto } \\
\text { de planos. } \\
\text { Ligar diferentes planos da organização e assegurar que os suprimentos estejam } \\
\text { disponíveis a fim de suprir a demanda existente. } \\
\text { Gerar um consenso entre planos operacionais e financeiros por meio de revisões } \\
\text { coordenadas, lideradas pela alta gerência a fim de integrar planos estratégicos, } \\
\text { operacionais e financeiros por um horizonte de tempo de médio prazo. } \\
\text { Criar alinhamento e integração na empresa; oferecer melhorias em nível operacional; } \\
\text { promover resultados com foco em uma única meta; gerar resultados concretos. }\end{array}$ & $\begin{array}{l}\text { Grimson \& Pyke (2007) } \\
\text { Tudorie \& Borangiu (2011) } \\
\text { Ivert \& Jonsson (2010) } \\
\text { Mellen et al. (2010) } \\
\text { VICS (2010) } \\
\text { Thomé et al. (2012b) }\end{array}$ \\
\hline
\end{tabular}

Fonte: Elaborado pelos autores. 
produtos, clientes e mercados também são possíveis por meio do S\&OP. Deste modo, o processo contribui para maior lucratividade, desempenho satisfatório da empresa e maior satisfação do cliente (Muzumdar \& Fontanella, 2006).

O processo proporciona um conjunto de planos de demanda que modelam planos de Vendas, Marketing e de Desenvolvimento de Novos Produtos, resultando, ainda, em uma gama de planos de suprimentos que delineiam as atividades de compras, suprimentos, manufatura e inventário. O S\&OP é direcionado pela previsão de demanda, levando em consideração que o equilíbrio entre os planos de demanda e de suprimentos devem ser guiados por metas e objetivos estratégicos. Métricas de desempenho são empregadas com o propósito de avaliar se as metas da empresa estão sendo atingidas e oferecer feedback ao processo de planejamento estratégico com o fim de analisar o progresso do processo. O processo tem como foco o planejamento contínuo, que é viável por meio de revisões mensais e ajustes contínuos dos planos, conforme as flutuações da demanda e do mercado ocorrem e da disponibilidade de recursos internos e do suprimento de materiais, bem como de serviços externos (Wallace, 2001).

O processo de $\mathrm{S} \& \mathrm{OP}$ vem trazer maior integração aos planos da empresa, envolvendo desde o nível estratégico até o operacional e modificando o fluxo de trabalho da organização. Tradicionalmente, as organizações são projetadas conforme um modelo linear, no qual as ações de planejamento de vendas e planejamento de operações ocorriam de forma sequencial, no qual a cultura de silos dominava. Neste modelo tradicional, não há consenso entre as áreas, pois as informações são apenas passadas por meio das áreas funcionais a fim de executar determinadas ações, sem que haja uma uniformidade de planos entre as áreas funcionais (Wallace, 2001).

No âmbito do S\&OP, a coordenação entre as áreas envolvidas é alcançada por meio de reuniões, nas quais planos conflituosos e questões pendentes são avaliadas à luz de materiais, tarefas, recursos financeiros e restrições de capacidade existentes, resultando em um conjunto de planos integrados (Feng et al., 2010). $\mathrm{O}$ antigo processo de Planejamento de Produção, que possuía intrinsecamente a abordagem de silos, determinava que Vendas e Marketing elaborasse a previsão de vendas e a entregasse ao planejamento de produção. O plano de produção resultante era, então, encaminhado ao programador mestre que era encarregado de dividi-lo em produtos individuais. O S\&OP, por sua vez, se mostra como um processo que demanda equipes multidisciplinares, ou seja, exige que as áreas de Vendas e Marketing, Operações, Finanças e Desenvolvimento do Produto trabalhem de modo conjunto para o desenvolvimento de uma gama integrada de planos que todos estes departamentos possam apoiar. Desta forma, possíveis recomendações elaboradas por meio do trabalho conjunto das áreas funcionais são apresentadas à equipe de S\&OP Executivo, que é responsável pela aprovação ou modificação destas recomendações. O processo resulta então, em um plano de ação autorizado para a organização como um todo (Wallace, 2001).

\section{Aspectos metodológicos}

Os aspectos metodológicos adotados ao longo da realização da pesquisa são apresentados na presente seção. $\mathrm{O}$ foco da pesquisa consiste em caracterizar a dinâmica do processo de S\&OP em três empresas que possuem como estratégia de resposta a demanda Make to Stock (MTS). Desta forma, na fase inicial da pesquisa foi realizada uma revisão de literatura sobre como acontece o processo de S\&OP. A revisão de literatura teve como intuito servir como embasamento teórico para a pesquisa, seguida por um estudo de caso para buscar incluir a realidade empírica de algumas empresas brasileiras na implantação do S\&OP. A abordagem é qualitativa, pois examina conceitos em termos dos seus significados e interpretações em um contexto específico de uma pesquisa, como sugerido por Ketokivi \& Choi (2014).

A condução da revisão de literatura foi realizada tendo como base os trabalhos de Tranfield et al. (2003), Tranfield et al. (2004) e Denyer \& Tranfield (2009). O passo inicial da revisão sistemática de literatura consistiu no acesso às bases de dados: Science Direct, Emerald e EBSCO. Estas bases de dados foram escolhidas, pois, conforme Thomé et al. (2012a), tais bases de dados possuem o maior escopo de artigos publicados, relacionados aos temas: Operações, Gestão de Organizações e Ciências Sociais no geral. A fim de caracterizar o processo de S\&OP, primariamente, com uma perspectiva mais genérica, para, assim, explorar a dinâmica do processo em âmbito mais estrito, as palavras-chaves utilizadas na busca foram: "S\&OP" e "Sales and Operations Planning", buscando-as em títulos de artigos, resumo e palavras-chaves. O string de busca utilizado foi: "S\&OP" OR "Sales and Operations Planning". O critério considerado para a inclusão dos artigos foi a consistência do artigo no que se relaciona aos constructos do S\&OP, incluindo os artigos que apresentassem uma base teórica consistente aos objetivos de pesquisa. Com relação ao critério utilizado para a exclusão de artigos, eliminaram-se os artigos que detalhavam temas associados ao S\&OP, porém sem abordar o processo de forma a promover embasamento teórico necessário para o desenvolvimento da pesquisa.

A busca, leitura e síntese dos artigos demorou cerca de 4 meses (entre abril e junho de 2013), sendo atualizadas no início de 2015. As buscas por artigos nas bases de dados Science Direct, Emerald e EBSCO retornaram 214 artigos, dos quais todos os resumos 
foram lidos. Dentro desta amostra, alguns artigos não se relacionavam ao tema e foram, portanto, excluídos. A partir deste primeiro filtro, restaram 118 artigos, que tiveram seus resumos, introduções e conclusões lidas. A partir desta análise, restaram 61 artigos, que foram lidos na íntegra e utilizados na revisão de literatura da dissertação de mestrado de uma das autoras da pesquisa. Dos 61 artigos finais, buscou-se utilizar neste artigo somente os artigos que respondessem ao objetivo pretendido. Literatura adicional foi incorporada à pesquisa, com o intuito de enriquecer a base conceitual teórica do trabalho. Buscou-se ainda, por Teses e Dissertações nacionais na Biblioteca Digital de Teses e Dissertações da USP e na Biblioteca da universidade em que foi realizado o trabalho. Periódicos nacionais na área de Administração da Produção também foram pesquisados, sendo buscados por meio da base de dados Scopus, a fim de encontrar artigos publicados nos periódicos "Gestão \& Produção" e "Produção", já que estes periódicos apresentam maior proximidade com a área de Administração da Produção e destaque no cenário nacional, retornando em um artigo que foi utilizado na pesquisa. Além disso, foram consultados os anais dos principais eventos na área de Operações em âmbito nacional: Simpoi, Enegep e Simpep, nos quais foram procurados artigos publicados nos últimos cinco anos. A busca retornou em cinco artigos que não foram utilizados, pois as informações trazidas pelos artigos encontrados em congressos nacionais já estavam presentes em outros artigos analisados previamente.

A coleta de dados para o estudo empírico foi realizada por meio de um estudo multicaso, pois ele permite que se realizem averiguações fundamentadas na realidade, investigando um determinado fenômeno e o contexto específico no qual está inserido. Este tipo de pesquisa é viável principalmente quando interações sobre o fenômeno estudado e seu contexto não estão claras (Yin, 2005). Na área de gestão de operações, este método tem sido apontando como relevante (Barratt et al., 2011; Ketokivi \& Choi, 2014), entretanto requer que os pesquisadores se preocupem com a transparência dos procedimentos e escolhas realizadas. Nesta pesquisa, houve a elaboração de um protocolo de pesquisa, sendo que a elaboração do questionário semiestruturado utilizado nos estudos de caso se baseou no objetivo da pesquisa. O questionário caracteriza-se como principal instrumento de coleta de dados da pesquisa. Além disso, o questionário foi submetido a um pré-teste, o qual foi revisado por cinco professores da área de Engenharia de Produção (experientes em metodologia e gestão de operações) de diferentes universidades (University of Tennessee, Universidade Federal de São Carlos, Universidade Federal de Uberlândia, Unesp/Bauru e Universidade Metodista de Piracicaba) em junho de 2013.
Estudos multicaso possibilitam a obtenção de resultados mais consistentes, já que proporcionam um estudo com uma perspectiva global, podendo, assim, serem realizados com maior robustez. Estudos multicaso apresentam, desta forma, maior expressividade, quando comparados a estudos de caso único. Tal fato é devido à replicação dos resultados da análise das características dos elementos constituintes da pesquisa, possibilitando a detecção de convergências e divergências entre eles, contribuindo assim para a solução do problema da pesquisa (Yin, 2005). Na seleção dos casos a serem estudados, adotaram-se como critério: empresas manufatureiras de bens de grande porte, deste modo, é possível obter maior variedade de constructos coletados em campo, sendo que empresas de grande porte apresentam maior complexidade (Massey \& Dawes, 2001; Paiva, 2010); organizações com sede no Estado de São Paulo, por questão de facilidade de acesso; organizações com a estratégia de orientação à demanda Make to Stock (MTS), pois os processos destas empresas são fundamentados principalmente na previsão da demanda, havendo maior grau de incerteza quanto à demanda do período (Pires, 1995). A maior relevância dos sistemas Make to Stock (MTS) pode ser explicada pelo fato de que empresas com estratégia Make to Order (MTO), que produzem sob encomenda, trabalham com pedidos firmes em carteira, portanto sua gestão é realizada de forma mais fácil. Com relação às empresas que seguem a estratégia Make to Stock (MTS), toda a gestão é baseada em previsão de vendas, estando sujeitas a todas as desvantagens e riscos associados a previsões (Pires \& Musetti, 2000). Desta forma, empresas com estratégia MTS foram selecionadas, já que a previsão de demanda é um dos principais recursos de entrada do S\&OP.

A coleta de dados foi realizada entre setembro de 2013 e fevereiro de 2014, tendo uma duração de, no mínimo, 1 hora a 1 hora e meia. No total, foram entrevistados 13 colaboradores das três organizações pesquisadas. A natureza semiestruturada dos questionários possibilitou ao entrevistado discursar mais livremente sobre o tema em pauta, podendo revelar dados adicionais e enriquecendo o processo de coleta de dados. Todas as entrevistas foram conduzidas de forma presencial, com os profissionais envolvidos com o processo de S\&OP, de cargos pertencentes a diversas áreas funcionais de três empresas de grande porte. Depois das entrevistas serem realizadas, foram transcritas e codificadas em categorias para que fosse possível sua quantificação. A escolha das pessoas a serem entrevistadas se deu em virtude de natureza interfuncional do processo de $\mathrm{S} \& \mathrm{OP}$, que demanda a participação de colaboradores de várias áreas (Thomé et al., 2012a,b). O Quadro 2 sintetiza 
as principais características das empresas envolvidas na pesquisa e atribui a cada uma um nome fictício.

No tratamento dos dados, foi utilizada a análise de conteúdo (Bardin, 1977). As entrevistas foram todas gravadas e transcritas. Em seguida, realizou-se a construção de um codebook que foi empregado no software QDA Mine. A categorização contida no codebook abrangeu as unidades de registro em categorias que foram identificadas a partir da revisão de literatura e seus principais pontos, segundo o escopo da pesquisa, primeiro caso a caso e depois uma análise intercasos. A análise dos resultados foi possível por meio da interpretação das informações obtidas em campo, com o auxílio do software QDA Miner e do Microsoft Excel. O software em questão foi usado principalmente para a análise dos casos individuais, sendo que na análise intercasos priorizou-se o Microsoft Excel. As categorias analisadas foram segregadas e analisadas de forma descritiva, em primeiro momento, e, posteriormente, de modo interpretativo, com base na literatura sobre S\&OP. A partir da geração das categorias no software, foi possível identificar subcategorias.

\section{Apresentação e discussão dos resultados}

No presente item, serão apresentados os resultados obtidos nas organizações pesquisadas caso a caso e, adiante, será apresentada a análise intercasos.

\subsection{MAQUIP}

O processo possuiu como principal motivador a necessidade de alinhar as informações, pois, antes de sua implantação, eram desconectadas. Além disso, a empresa entendeu que o processo de S\&OP a

Quadro 2. Características das empresas pesquisadas.

\begin{tabular}{|c|c|c|c|c|}
\hline Caso & Características gerais & $\begin{array}{l}\text { Quantidade de } \\
\text { funcionários }\end{array}$ & $\begin{array}{l}\text { Ano de adoção } \\
\text { do S\&OP }\end{array}$ & Entrevistados \\
\hline MAQUIP & $\begin{array}{l}\text { Empresa norte-americana, com } \\
\text { subsidiárias em diversos países. } \\
\text { É líder no setor de maquinário de } \\
\text { construção e mineração. Possui } \\
\text { concorrentes diretos, inclusive no } \\
\text { Brasil. Encontra-se em um mercado } \\
\text { de alta volatilidade, e precisa se } \\
\text { adaptar rapidamente às mudanças, } \\
\text { pois grandes eventos no setor de } \\
\text { construção civil podem influenciar a } \\
\text { sua demanda. }\end{array}$ & 4.500 & $\begin{array}{l}\text { Implantado em } \\
\text { 1997, alterado } \\
\text { em 2007. Contou } \\
\text { com auxílio de } \\
\text { consultoria. }\end{array}$ & $\begin{array}{l}\text { Diretor de Supply } \\
\text { Chain (DSC X), } \\
\text { Gerente de Estratégia } \\
\text { (GE X), Gerente de } \\
\text { Produto (GP X) e } \\
\text { Gerente de Sistemas de } \\
\text { Qualidade e Operações } \\
\text { (GSQO X). }\end{array}$ \\
\hline MAGRICO & $\begin{array}{l}\text { Empresa brasileira com plantas no } \\
\text { Brasil e Tailândia. É destaque no setor } \\
\text { de maquinário agrícola e exporta para } \\
\text { mais de } 60 \text { países. Alguns dos insumos } \\
\text { utilizados pela empresa, tais como } \\
\text { a fundição e a usinagem de peças, } \\
\text { são verticalizados, sendo que tais } \\
\text { matérias-primas são manufaturadas } \\
\text { na própria organização. Mercado } \\
\text { sazonal, influenciado por safras, clima } \\
\text { e política. }\end{array}$ & 3.000 & $\begin{array}{l}\text { Iniciado em } 2010 \text {, } \\
\text { pelos gestores } \\
\text { da empresa sem } \\
\text { ajuda externa. }\end{array}$ & $\begin{array}{l}\text { Gerente de Operações } \\
\text { (GO Y), Gerente } \\
\text { de Planejamento e } \\
\text { Controle de Produção } \\
\text { (GPCP Y), Gerente } \\
\text { de Vendas (GV Y) e } \\
\text { Assistente de Vendas } \\
\text { (AV Y). }\end{array}$ \\
\hline MATESCO & $\begin{array}{l}\text { Empresa europeia, com plantas } \\
\text { produtivas em países diversos e } \\
\text { exporta para mais de } 70 \text { países. Tem } \\
\text { destaque em seu setor de atuação, } \\
\text { sendo líder mundial do segmento. } \\
\text { Principais produtos ofertados pela } \\
\text { empresa: lápis de madeira, canetas, } \\
\text { marcadores, lapiseiras e grafites, } \\
\text { dentre outros. A organização se } \\
\text { encontra em um mercado de alta } \\
\text { sazonalidade (ocasionada pelo período } \\
\text { letivo das escolas brasileiras). }\end{array}$ & 2.500 & $\begin{array}{l}\text { Implantado em } \\
\text { fevereiro de } \\
\text { 2012, sendo que } \\
\text { a Gerência de } \\
\text { S\&OP foi criada } \\
\text { em junho. Houve } \\
\text { uma tentativa } \\
\text { de implantação } \\
\text { anterior em } 2008 \text {. }\end{array}$ & $\begin{array}{l}\text { Gerente de S\&OP } \\
\text { (GSOP Z), Especialista } \\
\text { de S\&OP (ESOP Z), } \\
\text { Coordenador de S\&OP } \\
\text { (CSOP Z), Supervisor } \\
\text { de Planejamento de } \\
\text { Produção (SPP Z) } \\
\text { e a Gerente de } \\
\text { Planejamento de } \\
\text { Vendas (GPV Z). }\end{array}$ \\
\hline
\end{tabular}


faria evoluir como um todo, ajudando-a a estudar as dificuldades e características do mercado local, auxiliando-a a desenvolver possíveis soluções para os problemas enfrentados. O alinhamento com as metas estratégicas da empresa precisa, de acordo com entrevistados, ser realizado de modo eficaz, já que a empresa apresenta uma complexidade na condução de seu gerenciamento, sendo que o gerenciamento do fluxo de informações proporcionado pelo S\&OP é capaz de auxiliar a organização a atingir tais objetivos. Os objetivos da empresa, quanto ao S\&OP, eram: melhorar a comunicação entre as áreas funcionais e otimizar o planejamento de capacidade.

Por meio das entrevistas realizadas, constatou-se que, antes da implantação do processo de S\&OP, o departamento de Marketing e Vendas recebia os relatórios e pedidos de produtos decorrentes da demanda externa. Estas informações alimentavam os sistemas da organização e, assim, avaliava-se o impacto gerado pela demanda. Desta forma, as informações eram passadas imediatamente para a cadeia de fornecedores que, por sua vez, disparam suas respectivas ações em resposta às informações passadas pela empresa, fato que impactava as demandas estipuladas. Antes do S\&OP, a comunicação era muito fragmentada, sendo que havia pouca troca de informações entre as áreas funcionais. A partir da implementação do S\&OP, o procedimento de receber e alimentar os sistemas com as demandas geradas só é realizado a partir do $11^{\circ}$ dia útil. Agora, a empresa pode processar a demanda e colocá-la no MRP e só então essas informações são passadas para sua cadeia de fornecimento. Quanto à capacidade instalada, anteriormente à implementação do processo de S\&OP, não havia visibilidade com antecedência, perdendo-se oportunidades pela falta de planejamento. A gestão da capacidade era muito mais fragmentada, havendo um planejamento de capacidade de máquina propriamente dito e não um planejamento mais abrangente.

Os principais resultados obtidos pela Maquip após 17 anos de implantação foram: a melhoria da comunicação entre as áreas funcionais e a otimização do planejamento da capacidade. Observa-se que após a implantação a Maquip realiza um planejamento com horizonte de cinco anos. Este planejamento leva em consideração um determinado mix de produtos que a organização almeja ter no futuro, planejando de forma antecipada, com a introdução de novos produtos de forma mais controlada. Realiza-se, em seguida, a reorganização de layout para se preparar para uma demanda futura, sendo comunicado com antecedência de 18 a 24 meses para toda a cadeia de fornecimento, a fim de promover maior previsibilidade de investimento para a cadeia. Assim, a partir da implementação do S\&OP, os processos de Gestão da Demanda e da Gestão da Capacidade começaram a trabalhar de forma mais estruturada, conforme fala de um dos entrevistados a seguir. Os principais desafios foram: fazer com todos avaliassem os impactos que estavam sendo gerados nas demais áreas e o reconhecimento da interdependência funcional. Foi mencionada, como desafio, também, a busca por conciliar demanda e suprimentos, tanto de forma interna à empresa, quanto na cadeia de suprimentos.

\begin{abstract}
We switched from supply driven to demand driven, that were basically inventory. In order for us to manage our inventory, we just can't produce and then plan to sell it. We needed to estimate what we were going to sell then produce that, and we are much more accurate doing it that way than the previous way. (DSC X - entrevista concedida em inglês).
\end{abstract}

\subsection{MAGRICO}

Começou-se a estudar o processo na organização no ano de 2007 e se instituiu um processo aproximadamente nos moldes do S\&OP. Desta forma, criou-se uma equipe multifuncional, com participantes de várias áreas funcionais como Tecnologia de Informação, Marketing, Vendas, Diretoria Industrial e Diretoria de Marketing. A partir deste ponto, todos os participantes foram instruídos a entender o processo, a fim de que ele fosse implantado. Para tal, alguns livros sobre o assunto foram estudados e estabeleceu-se o processo de S\&OP. Uma motivação para a implantação do S\&OP foi ele auxiliar no desenvolvimento da capacidade de alinhamento das estratégias, tornando mais fácil a construção de planos táticos a partir das estratégias na visão macro. Outra motivação era a possibilidade de ganhos a serem obtidos na área de planejamento, pois percebia-se que o S\&OP ajudaria a tornar mais eficientes os processos além de integrar a empresa. Outra motivação para a implantação do S\&OP foi a possibilidade de oferecer um planejamento mais detalhado para a empresa, além da necessidade de melhor utilização dos ativos. Os principais objetivos para a implementação do processo de S\&OP foram a melhoria do planejamento da capacidade, maior alinhamento entre demanda e suprimentos e otimização do planejamento da empresa. Antes da implantação do S\&OP, a Gestão da Capacidade era realizada de forma simples, não era feito o controle efetivo da capacidade buscando a visualização dos volumes. As necessidades de materiais eram calculadas por meio do MRP e a Gestão da Demanda e a Gestão da Capacidade não eram efetivas, simplesmente os dados da demanda eram colocados no sistema, independentemente de haver capacidade para produzir ou não. Ou seja, não existia alinhamento entre Vendas e Produção, nem colaboração das partes interessadas e consenso entre as áreas. Não havendo um número único, em alguns momentos, a empresa sofria ora 
com falta de entregas, ora com estoques elevados, impactando a cadeia como um todo.

Os processos de planejamento existentes antes da implantação do S\&OP eram executados sem periodicidade definida. Depois da implantação, todo mês o processo de S\&OP é rodado, sendo baseado nas metas estratégicas da empresa. Dispõem-se, nos dias atuais, de informações da demanda de todo o mês, havendo apenas pequenas variações que ocorrem normalmente dentro do processo estratégico, já que ele se renova mensalmente. Conhece-se também a demanda para os próximos 12 meses, e, desta forma, o processo ocorre naturalmente, com pequenos ajustes apenas e sem mudança de calendário. Dentre os principais resultados, estão o alinhamento entre as metas estratégicas e operacionais e maior assertividade na previsão de demanda. Os desafios enfrentados pela empresa englobam a construção de consenso entre as áreas funcionais a fim de realizar o planejamento da capacidade de modo otimizado e promover visibilidade das informações a todos os envolvidos para equilibrar demanda e suprimentos de forma interna à empresa.

Mesmo antes da implantação do S\&OP já havia um processo de planejamento estruturado, porém sua implementação trouxe maior robustez, tanto para a Gestão da Demanda, quanto para a Gestão da Capacidade (GV Y). O planejamento de capacidade era muito superficial, nós não tínhamos pré-acordos, e tampouco os limites claros de quais eram os horizontes de congelamento para cada família. Uma das coisas principais que o S\&OP elevou foi o alinhamento entre as áreas (GPCP Y).

\subsection{MATESCO}

Nesta empresa, no ano de 2008, houve uma tentativa de implantação do processo de S\&OP realizada pelos colaboradores da organização. Porém, o projeto não foi levado adiante na época, ressurgindo em 2012, com a colaboração de uma consultoria externa que a auxiliou a desenhar os processos e definir os indicadores de desempenho necessários. O principal responsável pela implantação do processo foi o Gerente de S\&OP, porém todas as áreas envolvidas no processo participaram ativamente de sua implantação.

Os principais motivos que incentivaram a implantação do S\&OP foram a necessidade de entender e elevar o nível de serviço ao cliente, redução de inventário, melhoria de capital de giro e a necessidade de diminuir a dificuldade de realizar a previsão da demanda. Além destes fatores, havia dificuldade por parte da área de Compras e de Produção em reagir à demanda, pois havia muita oscilação no mercado. $\mathrm{O}$ atendimento das necessidades do mercado e a otimização dos recursos da empresa também foram fatores motivadores para implantação do S\&OP, além da necessidade da criação de um processo único a fim de haver um só objetivo consistente a todas as áreas da empresa, buscando garantir imparcialidade na tomada de decisão.

Os principais objetivos eram obter maior periodicidade no planejamento, desenvolver planos mais assertivos, otimizar o planejamento das unidades de negócio e promover maior equilíbrio entre demanda e suprimentos. Antes da implementação do S\&OP, a gestão da demanda e da capacidade eram feitas com menor periodicidade, sendo que as revisões eram realizadas a cada 3 ou 4 meses, gerando planos que não estavam de acordo com a realidade. Além disso, o planejamento de Marketing e de Vendas era feito de forma única e não sequencial, havendo pouca participação da área de Vendas, no qual o planejamento era direcionado exclusivamente para o cumprimento de metas. Para alguns produtos originados na mesma fábrica, também era necessário realizar a revisão do planejamento duas vezes, uma para o mercado interno e outra para o mercado externo, não havendo sincronismo entre as unidades de negócio. Atualmente há padronização, todas as fábricas seguem um único calendário. Já para a gestão da capacidade, quando o PCP terminava suas análises de restrição de capacidade, apenas enviava uma planilha por e-mail ao departamento de Vendas, informando as restrições, não havendo discussão conjunta alguma. Desta forma, as restrições identificadas em nada interferiam no plano de Vendas, pois não havia uma orientação de quais produtos deveriam ser vendidos.

Depois da implantação, na reunião de consenso, há uma lista de todos os produtos que necessitam ser vendidos e de outros produtos que estão em falta no estoque, sendo que tudo isso é passado ao pessoal de Venda. A Gestão da Demanda era realizada a partir de um histórico que era colocado em uma planilha do Excel, os Gerentes de Marketing definiam para as grandes famílias de produtos quais seriam os volumes a serem vendidos e este plano se tornava meta para os vendedores. A Matesco implantou uma ferramenta estatística para melhorar a previsão de vendas e os vendedores também participam da confecção dos números e da revisão mensal, havendo, deste modo, uma visão comercial.

Como resultado, hoje se observa que há maior comunicação entre as áreas de Marketing e de Vendas, sendo que as informações fluem de forma satisfatória entre os departamentos, fazendo com que os dados disponíveis em campo retornem para a empresa. Anteriormente à implementação do $\mathrm{S} \& \mathrm{OP}$ havia um processo definido de como transformar a demanda em plano de produção, porém, a diferença é que, atualmente, com o S\&OP, as restrições de capacidade encontradas retornam para a área Comercial, consolida-se e divulga-se um novo plano. No processo de Gestão 
da Capacidade, de acordo com os entrevistados, ocorreram poucas mudanças quanto ao modo de se trabalhar com a previsão de demanda, pois ainda se está aprendendo a trabalhar com o novo software que foi implantado. O principal papel deste software é de tornar o processo mais rápido, promovendo respostas mais ágeis, análises mais conclusivas e auxiliando a organização a enxergar mais detalhes. Outro ponto em que houve mudanças foi a devolução da análise da Gestão da Demanda, feita pela área de Produção da empresa, sendo que esta análise, hoje conta com muito mais informações, é mais pautada e possui mais detalhes de tudo o que ocorre nos processos produtivos. Desta forma, atualmente, há muito mais interface entre as áreas, conforme fala de alguns entrevistados. Os principais resultados foram melhoria da comunicação entre as áreas e maior integração dos planos de demanda e de suprimentos. Os principais desafios foram o empenho da empresa a fim de alinhar demanda e suprimentos, realizar o planejamento da capacidade de forma satisfatória e melhorar comunicação e visibilidade entre as áreas.

Antes nem todos os departamentos participavam ativamente eagora com oprocesso tudo melhorou.(CSOPZ) A gente tem na hora da devolução da análise da gestão da demanda, informações mais pautadas é o que hoje a gente passa muito mais para outra área são os detalhes de tudo o que está acontecendo dentro da fábrica, então, por exemplo, tem muito mais detalhe. Antes a outra área não tinha tanta visão do que acontecia, hoje tem muito mais interfaces. (SPP Z)

\subsection{Intercasos}

Neste item é realizada uma análise intercasos a partir dos dados das três empresas pesquisadas.

\subsubsection{Principais resultados obtidos e desafios enfrentados}

Atualmente, com o processo de S\&OP, percebe-se que, nas três organizações pesquisadas, tanto os processos de Gestão da Demanda, quanto da Gestão da Capacidade estão mais alinhados, sendo que tanto o volume de demanda, quanto as restrições de capacidade são avaliadas e consideradas, confirmando os achados na literatura (Wallace, 2004, 2006; Lapide, 2005; Vollmann et al., 2006; Corrêa et al., 2007; Ling \& Coldrick, 2009; Krajewski et al., 2009; Chen-Ritzo et al., 2010; Godsell et al. , 2010; VICS, 2010; Thomé et al., 2012a).

Os resultados alcançados pelas organizações são condizentes com os objetivos iniciais estabelecidos e com os motivadores para a implementação do processo de S\&OP. A organização Maquip teve como objetivo a melhoria da comunicação entre as áreas funcionais e do planejamento de capacidade. Depois da implementação do S\&OP, a organização foi capaz de obter melhorias na comunicação entre as áreas funcionais e otimizar o planejamento da capacidade. A empresa Magrico buscava a melhoria do planejamento da capacidade, bem como do planejamento da empresa e maior equilíbrio entre demanda e suprimentos. Os resultados alcançados foram: a obtenção de assertividade na previsão de demanda e maior ajustamento entre as metas estratégicas e operacionais da organização. Por fim, a empresa Matesco tinha como objetivos harmonizar demanda e suprimentos, promover maior periodicidade no planejamento da empresa, realizar o planejamento das unidades de negócio de forma satisfatória e desenvolver planos mais assertivos. Os resultados alcançados pelo processo foram maior equilíbrio entre demanda e suprimentos e melhoria na comunicação entre as áreas funcionais.

Os desafios enfrentados pela empresa Maquip foram: conciliar demanda e suprimentos e realizar a avaliação dos impactos gerados nas outras áreas funcionais. As empresas Magrico e Matesco enfrentaram dificuldades em realizar o planejamento da capacidade e para promover o equilíbrio entre demanda e suprimentos. Desta forma, os principais desafios notados nas três organizações pesquisadas foram: conciliar demanda e suprimentos de forma interna, exercer o controle da capacidade e avaliação dos impactos gerados pelas áreas funcionais. A implementação do processo de $\mathrm{S} \& \mathrm{OP}$ foi primordial para que tais desafios fossem superados. Um último desafio enfrentado foi a dificuldade com a própria natureza do processo, a necessidade de maior empenho por parte dos representantes da área de Operação na condução do processo. Este fato pode ser devido a situações rotineiras vivenciadas nas empresas, que se caracterizam pela própria natureza do processo que visa conciliar demanda com suprimentos. A área de Operações é geralmente cobrada de forma diferente acerca dos resultados esperados, quando comparada a outras áreas participantes do processo, tais como Vendas e Marketing, que são cobradas de modo mais brando. Tais constatações foram baseadas principalmente nas observações realizadas em campo, as quais foram essenciais para a coleta de dados. Este fenômeno vai de encontro com os achados na literatura. Conforme Wallace (2001), geralmente a equipe de operações é cobrada para atingir as metas estipuladas de produção, enquanto a equipe de vendas raramente é cobrada a ajustar planos de vendas. Ainda, a responsabilidade da área de Operações em sincronizar demanda e suprimentos pode ser exemplificada, segundo Slack et al. (2001). Os autores afirmam que, para as empresas conciliarem, de um lado as necessidades do mercado (representadas pela demanda) e, de outro lado, as capacidades da operação/suprimentos, a organização deve ser capaz de integrar os recursos de produção para que ela possa oferecer seus produtos ao 
consumidor. Desta forma, esta responsabilidade recai no planejamento e controle da produção (atividade típica de Operações) que têm a responsabilidade de conciliar estas duas atividades.

\section{Conclusões}

A contribuição trazida pela pesquisa reside no fato de o trabalho detalhar a dinâmica do processo de S\&OP, contrastando com o que motivou as empresas pesquisadas a implementar o processo, desafios e resultados obtidos. Outra contribuição da pesquisa foi preencher em parte uma lacuna empírica, já que o tema é pouco explorado em âmbito brasileiro, considerando estudos que tratem o assunto de forma empírica. Além disso, a maturidade de alguns processos gerenciais estudados é diferente dos estudos realizados em países seja da América do Norte, seja da Europa ou Ásia.

Os principais desafios encontrados nas empresas pesquisadas foram comunicação fragmentada e a dificuldade de construir visibilidade da gestão de capacidade. Estes desafios foram superados de diferentes maneiras pelas empresas, pois, em cada uma, existia uma cultura mais ou menos orientada para o mercado. Verificou-se que, para sincronizar demanda e suprimentos, há necessidade de que processos diferentes sejam redesenhados, assim como a periodicidade de reuniões deve ser alterada e deve haver a inclusão de funções que nem sempre eram consideradas no planejamento anteriormente.

Como resultado, observou-se melhoria na comunicação, otimização do planejamento de capacidades, maior assertividade na previsão de vendas. Desde que o S\&OP seja devidamente implementado e conduzido, considerando as particularidades de cada empresa, ele pode contribuir para a superação de vários dos desafios mencionados. O processo pode promover maior alinhamento entre as faces de demanda e de suprimentos na organização, sendo que tanto o volume de demanda, quanto as restrições de capacidade são avaliadas e consideradas, oferecendo um consenso único sobre os planos desenvolvidos. $\mathrm{O}$ maior conhecimento do mercado pelas áreas funcionais ligadas à face suprimentos bem como a melhoria da comunicação entre as áreas funcionais também são aspectos que merecem destaque. Já que o mercado nacional é mais sujeito a oscilações, convém que a empresa esteja a par das situações vivenciadas em seu ambiente. Ademais, empresas somente poderão alcançar os resultados do S\&OP caso os desafios inerentes à implementação do processo sejam enfrentados.

Diversas opções de estudos futuros são projetadas ao fim deste artigo. Podem ser efetuados estudos que relacionem o nível de maturidade com os fatores críticos de sucesso, procurando investigar a existência de relacionamentos entre ambos. Mostram-se necessárias também pesquisas que promovam o entendimento da forma pela qual o $\mathrm{S} \& \mathrm{OP}$ colabora para a integração interfuncional e outras ampliem o estudo do processo S\&OP e seu impacto no relacionamento com fornecedores e clientes. Por fim, seriam bem-vindos estudos que explorassem o processo de implantação do S\&OP por meio de uma survey, analisando a implantação do processo sob uma perspectiva quantitativa e estudos que explorem o processo de S\&OP no setor de serviços.

\section{Agradecimentos}

As autoras agradecem o suporte financeiro à CAPES (Bolsa de mestrado da $1^{\text {a }}$ autora) e à Fundação de Amparo à Pesquisa do Estado de São Paulo - FAPESP (Processo n²011/06008-8).

\section{Referências}

Association for Operations Management - APICS. (2009) Sales and Operations Planning: the secret to world class supply chain. Apics dinner meeting. Recuperado em 26 de fevereiro de 2013, de http:/www.apics-fraservalley.org

Bardin, L. (1977). Análise de conteúdo. Lisboa: Edições 70.

Barratt, M., Choi, T. Y., \& Li, M. (2011). Qualitative case studies in operations management: Trends, research outcomes, and future research implications. Journal of Operations Management, 29(4), 329-342.

Basu, R. (2001). New criteria of performance management: a transition from enterprise to collaborative supply chain. Measuring Business Excellence, 5(4), 7-12.

Baumann, F. (2010). The shelf-connected supply chain: strategically linking CPFR with S\&OP at the executive level. Journal of Business Forecasting, 29(4), 21-27.

Bower, P. (2005). Twelve most common threats to sales and operations planning process. Journal of Business Forecasting, 24(3), 4-14.

Boyer, J. E., Jr. (2009). Ten proven steps to successful S\&OP. Journal of Business Forecasting, 28(1), 4-10.

Bremer, C. F., Azevedo, C. R., \& Matheus, L. F. (2008a). $\mathrm{O}$ retrato do processo de Sales \& Operations Planning (S\&OP) no Brasil. Parte 1. Revista Mundo Logística, (5), 68 .

Bremer, C. F., Azevedo, C. R., \& Matheus, L. F. (2008b). $\mathrm{O}$ retrato do processo de Sales \& Operations Planning (S\&OP) no Brasil. Parte 2. Revista Mundo Logística, (6), 10 .

Burrows, R. P. (2007). Demand driven S\&OP: a sharp departure from the traditional ERP approach. Ohio: On Point Group.

Cecere, L., Barrett, J., \& Mooraj, H. (2009). Sales and Operations Planning: transformation from tradition. Industry value chain strategies. Boston: AMR Research. 
Chen-Ritzo, C. H., Ervolina, T., \& Harrison, T. P. (2010). GUPTA, B. Sales and operations planning in systems with order configuration uncertainty. European Journal of Operational Research, 205(3), 604-614.

Corrêa, H. L., Gianesi, I. G. N., \& Caon, M. (2007). Planejamento, programação e controle da produção (5. ed.). São Paulo: Atlas.

Cox, J. F., \& Blackstone, J. H. (2002). APICS Dictionary. Alexandria: APICS.

Denyer, D., \& Tranfield, D. (2009). Producing a systematic review. In D. A. Buchanan \& A. Bryman (Eds.), The sage handbook of organizational research methods ( $\mathrm{p}$. 671-689). London: Sage Publications.

Dougherty, J., \& Gray, C. (2006). Sales \& Operations Planning: best pratices, partners for excellence. Belmont: Trafford Publishing.

Elbaum, S. (2005). Best practices in S\&OP: a benchmark report. Boston: Aberdeen Group. Recuperado em 18 de março de 2013, de www.aberdeen.com

Esper, T. L., Ellinger, A. E., Stank, T. P., Flint, D. J., \& Moon, M. (2010). Demand and Supply Integration: a conceptual framework of value creation through knowledge management. Journal of the Academy of Marketing Science, 38(5), 1-15.

Feng, Y., D'Amours, S., \& Beauregard, R. (2008). The value of sales and operations planning in oriented stand board industry with make to order manufacturing system: cross functional integration under deterministic demand and spot market resource. International Journal of Production Economics, 115(1), 189-209.

Feng, Y., D'Amours, S., \& Beauregard, R. (2010). Simulation and performance evaluation of partially and fully integrated Sales and operations planning. International Journal of Production Research, 48(19), 5859-5883.

Gianesi, I. N. (1998). Implementing manufacturing strategy through strategic production planning. International Journal of Operations \& Production Management, 18(3), 286-299.

Gilmore, D. (2005) Integrated supply chain require effective sales and operations planning. Springboro: Supply Chain Digest. Recuperado em 13 de maio de 2013, de www.scdigest.com.

Godsell, J., Birtwistle, A., \& Van Hoek, R. (2010). Building the supply chain to enable business alignment: lessons from British American Tobacco (BAT). Supply Chain Management: an International Journal, 15(1), 10-15.

Grimson, J. A., \& Pyke, D. F. (2007). Sales and operations planning: an exploratory study and framework. The International Journal of Logistics Management, 18, 322-346.

Ivert, L. K., Dukovska-Popvska, I., Kaipia, R., Fredriksson, A., Dreyer, H. C., Johansson, M. I., Chabada, L., Damgaard, C. M., \& Tuomikangas, N. (2015). Sales and operations planning: responding to the needs of industrial food producers. Production Planning \& Control: The Management of Operations, 26(4), 280-295.

Ivert, L. K., \& Jonsson, P. (2010). The potential benefits of advanced planning and scheduling systems in sales and operations planning. Industrial Management \& Data Systems, 110(5), 659-681.

Ivert, L. K., \& Jonsson, P. (2014). When should advanced planning and scheduling systems be used in sales and operations planning? International Journal of Operations \& Production Management, 34(10), 1338-1362.

Ketokivi, M., \& Choi, T. (2014). Renaissance of case research as a scientific method. Journal of Operations Management, 32(5), 232-240.

Krajewski, L. J., Ritsman, L. P., \& Malhotra, M. K. (2009). Administração de produção e operações (8. ed.). São Paulo: Prentice Hall.

Lapide, L. (2011). S\&OP: the linchpin planning process. The Journal of Business Forecasting, 30(3), 4-5.

Lapide, L. (2004). Sales and operations planning part I: the process. The Journal of Business Forecasting, 23(3), 17-19.

Lapide, L. (2005). Sales and operations planning Part III: a diagnostic model. The Journal of Business Forecasting, 24(1), 13-16.

Ling, D., \& Coldrick, A. (2009) Breakthrough Sales and Operations Planning: how we develop the process. Recuperado em 12 de março de 2013, de www. lingcoldrick.com

Massey, G., \& Dawes, P. L. (2001). Integrating Marketing and Sales: The Frequency and Effectiveness of Methods Used in Australia and the United Kingdom. In Proceedings of the ANZMAC Conference. Auckland: ANZMAC.

Mellen, C., Allen, B., Prokopets, L., \& Putting, S. (2010). OP on the fast track. Supply Chain Management Review, 14(1), 40-45.

Moon, M. A. (2006). Breaking down barriers to forecast process improvement. The International Journal of Applied Forecasting, (4), 26-30.

Muzumdar, M., \& Fontanella, J. (2006). The secrets to S\&OP sucess. Supply Chain Management Review, 10(3), 34-41.

Olhager, J., Rudberg, M., \& Wikner, J. (2001). Long term capacity management: linking the perspectives from manufacturing strategies and sales and operations planning. International Journal of Production Economics, 69(2), 215-225.

Oliva, R., \& Watson, N. (2011). Cross-Functional Alignment in Supply Chain Planning: A Case Study of Sales and Operations Planning. Journal of Operations Management, 29(5), 434-448.

Oliva, R., \& Watson, N. (2009). Managing functional biases in organizational forecasts: A casestudy of consensus 
forecasting in supply chain planning. Production and Operations Management, (18), 138-151.

Paiva, E. L. (2010). Manufacturing and marketing integration from a cumulative capabilities perspective. International Journal of Production Economics, 126, 379-386.

Palmatier, G. E., \& Crum, C. (2010). A transition from sales and operations planning to integrated business planning (Oliver Wight whitepaper series). Oliver Wight Americas. Informative Guide on Industry Best Practices.

Pandim, F. J., Pereira, N. A., \& Politano, P. R. (2012). Modelo quantitativo para avaliação e melhoria de desempenho do processo de S\&OP baseado no diagnóstico e redução de falhas. Gestão \& Produção, 19(2), 361-375.

Pires, S. R. I. (1995). Gestão estratégica da produção. Piracicaba: Unimep.

Pires, S. R. I., \& Musetti, M. A. (2000). Logística integrada e gestão da cadeia de suprimentos. Produtos \& Serviços, (312), 65-76.

Santos, R. A. B. (2006). Sales and operations planning: uma maneira simples de obter ganhos com a integração interna. Revista Tecnologística, (127).

Sharp, B. W. (2006). An Investigation of the factors affecting successful sales and operations planning activities in the UK. (Dissertação de mestrado). School of Industrial and Manufaturing Science, Cranfield University, Bedfordshire.

Sheldon, D. H. (2006). World Class Sales \& Operations Planning: a guide to successful implementation and robust execution. Fort Lauderdale: J. Ross Publishing. Recuperado em 28 de fevereiro de 2012, de http:// books.google.com.br

Singh, M. K. (2010). What makes a winning S\&OP Program. Supply Chain Management Review, 14(3), 22-27.

Singhal, J., \& Singhal, K. (2007). Holt, Modigliani, Muth and Simon's work and its role in the reinassence and evolution of operations management. Journal of Operations Management, 25(2), 300-309.

Slack, N., Chambers, S., \& Johhston, R. (2001). Administração da produção (2. ed.). São Paulo: Atlas.

Tearnan, R. (2008) S\&OP Data Management: critical to a successful implementation. Recuperado em 29 de março de 2013, de http://www.oliverwight-americas.com

Thomé, A. M. T., Scavarda, L. F., \& Fernandez, N. (2012a). SCAVARDA, A. J. Sales and Operations Planning: A Research Synthesis. International Journal of Production Economics, 138(1), 1-13.

Thomé, A. M. T., Scavarda, L. F., \& Fernandez, N. (2012b). SCAVARDA, A. J. Sales and operations planning and the firm performance. International Journal of Productivity and Performance Management, 61(4), 359-381.

Thomé, A. M. T., Sousa, R. S., \& Carmo, L. F. R. R. S. (2014). The impact of sales and operations planning practices on manufacturing operational performance. International Journal of Production Research, 52(7), 2108-2121.

Tranfield, D., Denyer, D., Marcos, J., \& Burr, M. (2004). Co-producing management knowledge. Management Decision, 42(3-4).

Tranfield, D., Denyer, D., Smart, P. (2003). Towards a methodology for developing evidence-informed management knowledge by means of systematic review. British Journal of Management, 14(3),207-222.

Tudorie, C. R., \& Borangiu, T. (2011). Towards great challenge in sales and operation planning. In Proceeding of the IEEE 6th International Conference on Intelligent Data Acquisition and Advanced Computing Systems: Technology and Applications - IDAACS 2011 (Vol. 1).

Tuomikangas, N., \& Kaipia, R. (2014). A coordination framework for sales and operations planning (S\&OP): Synthesis from the literature. International Journal of Production Economics, 154, 243-262.

Viswanathan, N. (2011). Sales and Operations Planning: key enabler for the chief supply. Boston: Chain Officer, Aberdeen Group.

Vollmann, T. E., Berry, W. L., Whybark, D. C., \& Jacobs, F. R. (2006). Sistemas de Planejamento \& Controle da Produção: para gerenciamento da cadeia de suprimentos (5. ed.). Porto Alegre. The Bookman.

Voluntary Interindustry Commerce Solutions - VICS. (2010) Linking CPFR and S\&OP: a roadmap to integrated business planning. Recuperado em 14 de março de 2013, de http://vics.org

Wallace, T. F., \& Stahl, R. A. (2003). Planejamento moderno da produção (E. Toporcov, Trad.). São Paulo: IMAM.

Wallace, T. F. (2001). Planejamento de vendas e operações: guia prático (E. Toporcov, Trad.). São Paulo: IMAM.

Wallace, T. F. (2004). Sales and operations planning: the how-to handbook. Ohio: T. F. Wallace \& Company. Recuperado em 28 de fevereiro de 2013, de http:// books.google.com.br

Wallace, T. F. (2006). Forecasting and sales and operations planning: synergy in action. The Journal of Business Forecasting Methods \& Systems, 25(1), 16-36.

Wang, J. Z., \& Hsu, P. Y. (2010). Advanced Sales and operations planning based on integration of physical and financial flows. In Proceedings of the IEEE International Conference on Industrial Engineering and Engineering Management - IEEM (pp. 70-74). IEEE.

Wing, L., \& Perry, G. (2001). Toward twenty first century pharmaceutical sales and operations planning (p. 2026). Pharmaceutical Technology.

Yin, R. K. (2005). Estudo de caso: planejamento e métodos (3. ed.). Porto Alegre: Bookman. 
APÊNDICE 1. Protocolo de pesquisa

\section{Protocolo do Estudo de Caso}

\section{1) Objetivo da pesquisa}

O objetivo desta pesquisa é identificar os fatores para uma implantação bem-sucedida do processo de Sales and Operations Planning (S\&OP).

\section{2) Questão de pesquisa}

Quais são os fatores que caracterizam uma implementação bem-sucedida de S\&OP?

\section{3) Seleção das unidades de análise}

O critério de seleção das empresas em estudo na presente pesquisa são:

- Empresas que se encontram em fases distintas de implantação do processo de S\&OP, para que, desta forma, fosse possível desenvolver o entendimento sobre os fatores de sucesso para a implantação bem sucedida do S\&OP, por meio da detecção de elementos em comum entre os casos;

- Empresas de manufatura de bens de grande porte, deste modo, é possível obter maior variedade de constructos coletados em campo, sendo que empresas de grande porte apresentam maior complexidade (Massey \& Dawes, 2001; Paiva, 2010);

- Empresas localizadas no Estado de São Paulo, por questão de facilidade de acesso;

- Empresas com a estratégia de orientação à demanda Make to Stock (MTS), pois tais organizações fabricam produtos padronizados, fundamentados principalmente na previsão da demanda e apresentam maior grau de incerteza quanto à demanda do período (Pires, 1995). Assim, empresas com estratégia MTS foram selecionadas pelo fato da previsão de demanda se caracterizar como um dos principais recursos de entrada do S\&OP.

\section{4) Coleta de dados}

a) Empresas candidatas à pesquisa:

- Empresa A

- Empresa B

- Empresa C

b) Fontes de dados

- Entrevistas:

$\checkmark$ Contato inicial com a empresa por meio de e-mail/telefonema;

$\checkmark$ Seleção de pessoas de diferentes áreas funcionais que estejam envolvidas com o S\&OP há pelo menos 2 ciclos do processo;

$\checkmark$ Investigação do fenômeno de forma mais profunda com o coordenador do processo.

- Observação em campo:

c) Identificar todos os entrevistados;

d) Agendar as entrevistas;

e) Coletar os dados e analisá-los.

- Coleta de dados:

$\checkmark$ Apresentar os objetivos da pesquisa;

$\checkmark$ Dar ao entrevistado um exemplar do questionário que será aplicado (Apêndice 2);

$\checkmark$ Gravar as entrevistas (em média 1 hora por entrevista);

$\checkmark$ Fazer anotações durante a entrevista.

\section{5) Análise dos dados}

a) Codificação com base nos códigos derivados da literatura;

b) Análise dos códigos;

c) Análise intercasos;

d) Ligar a teoria encontrada na revisão de literatura com os resultados dos estudos de caso. 
APÊNDICE 2. Questionário

\section{Questionário aplicado na coleta de dados}

Empresa:

Observação: O nome da empresa será omitido do trabalho. Desde já, agradecemos sua colaboração! As questões apresentadas formam a base para discussão. Contudo, estas questões não se limitam ao escopo da discussão, caso outras informações importantes forem identificadas. Com sua permissão, gostaria de gravar a entrevista a fim de ajudar a validar as informações. A gravação será confidencial e somente a equipe de pesquisa terá acesso a ela.

Estudo de Caso - Caracterização dos Fatores para a implantação do Processo de Sales and Operations Planning (S\&OP)

\section{DADOS GERAIS:}

Nome do entrevistado:

Cargo atual na empresa / tempo no cargo:

Cargo anterior na empresa/ tempo no cargo:

\section{QUESTÕES SOBRE S\&OP}

1 - Histórico do processo de S\&OP na empresa:

a) Por favor, descreva o processo de S\&OP na empresa.

b) Por quanto tempo o processo de S\&OP vem sendo utilizando desta maneira?

c) Quais foram as motivações primárias para implantar o processo na empresa?

d) Explique de que forma o S\&OP está alinhado com as metas estratégicas da empresa?

e) Descreva o processo de implantação do S\&OP. Quem foram os envolvidos, tanto interna quanto externamente?

f) Foram necessárias novas habilidades/treinamentos/conhecimento/tecnologia na fase de implantação do S\&OP e para geri-lo atualmente?

2 - Quais são as áreas envolvidas no processo? De que nível hierárquico são os envolvidos? Quem lidera o processo de $\mathrm{S} \& \mathrm{OP}$ ?

a) Qual o papel da alta gerência no S\&OP?

b) Descreva o papel de Logística no S\&OP. Como esta área poderia contribuir mais efetivamente para o processo?

3 - Processos impactos

a) Como o processo de Gestão da Demanda era feito na empresa antes da implantação do S\&OP?

b) Como o processo de Gestão de Capacidade era feito na empresa antes da implementação do S\&OP?

4 - Você poderia discutir, por favor, qual o seu papel no processo de S\&OP, bem como o papel de sua área funcional?

5 - Como o S\&OP funciona na companhia? Descreva as etapas do processo, por favor.

6 - Quais foram os benefícios obtidos por meio do S\&OP?

a) Quais foram as principais barreiras enfrentadas na implantação do S\&OP?

b) Discuta sobre o grau de dificuldade da implantação do S\&OP na empresa.

c) Como você considera o custo da implantação do S\&OP (alto, médio, baixo)? A companhia estimou os custos da implantação?

d) Como essas dificuldades foram superadas?

7 - O processo conta com auxílio de software ou de ferramentas de tecnologia de informação? Descreva e fale do papel/importância delas.

8 - Existem métricas de avaliação de desempenho desenvolvidas pelo processo? Descreva.

9 - Estas métricas avaliam áreas individualmente ou resultados produzidos em conjunto?

10 - Quais são os objetivos atuais da empresa com relação ao S\&OP?

11 - Quais são as expectativas para o futuro com relação ao S\&OP?

12 - De que forma o S\&OP contribui para a integração das áreas funcionais da empresa? 
13 - Quais fatores você considera como críticos para a implantação bem-sucedida do S\&OP? 14 - Houve maior integração interfuncional entre as áreas após a implantação do S\&OP?

a) Como você descreve o processo de S\&OP em termos de maturidade? Há oportunidades para melhorias, ou você o considera relativamente estático?

15 - Mais algum comentário que você gostaria de fazer sobre o processo de S\&OP na empresa? 